\title{
Electronic Structure and Magnetic Properties of Ferromagnetic Shape Memory Alloys CoNiAl*1
}

\author{
Shinpei Fujii ${ }^{1}$, Shoji Ishida ${ }^{1}$ and Setsuro Asano ${ }^{2, * 2}$ \\ ${ }^{1}$ Department of Physics, Faculty of Science, Kagoshima University, Kagoshima 890-0065, Japan \\ ${ }^{2}$ Institute of Physics, College of Arts and Sciences, University of Tokyo, Tokyo 153-0041, Japan
}

\begin{abstract}
To expand our knowledge of electronic structures and magnetic properties for ferromagnetic shape memory Co-Ni-Al alloys, which undergo a thermoelastic martensitic transformation from a $B 2$ to an $L 1_{0}$ structure, we have performed first-principles band calculations for the composition of $\mathrm{Co}: \mathrm{Ni}: \mathrm{Al}=1: 1: 1$, i.e., $\left(\mathrm{Co}_{1 / 3} \mathrm{Ni}_{2 / 3}\right)\left(\mathrm{Co}_{1 / 3} \mathrm{Al}_{2 / 3}\right)$ and $\left(\mathrm{Ni}_{1 / 3} \mathrm{Co}_{2 / 3}\right)\left(\mathrm{Ni}_{1 / 3} \mathrm{Al}_{2 / 3}\right)$. Their electronic structures have been calculated for the supercell structure, including a cubic-tetragonal distortion and a spin polarization. The obtained total energy indicates that $\left(\mathrm{Co}_{1 / 3} \mathrm{Ni}_{2 / 3}\right)\left(\mathrm{Co}_{1 / 3} \mathrm{Al}_{2 / 3}\right)$ may be more appropriate than $\left(\mathrm{Ni}_{1 / 3} \mathrm{Co}_{2 / 3}\right)\left(\mathrm{Ni}_{1 / 3} \mathrm{Al}_{2 / 3}\right)$. In the paramagnetic state of $\left(\mathrm{Co}_{1 / 3} \mathrm{Ni}_{2 / 3}\right)\left(\mathrm{Co}_{1 / 3} \mathrm{Al}_{2 / 3}\right)$, it has been found that the transformation from the $B 2$ to the $L 1_{0}$ structure comes from the change of the environment of the $\mathrm{Co}$ atoms $(\mathrm{Co}[2 \mathrm{e}])$ on the original $\mathrm{Al}$ sites and, other $\mathrm{Co}$ and $\mathrm{Ni}$ atoms. These features are reflected in their $d$-orbital density of states (DOS). The Co[2e] atoms also play an important role in the magnetic transition between the paramagnetic and ferromagnetic states. The Co[2e] atoms carry magnetic moments corresponding to those of fcc $\mathrm{Co}$ (hcp $\mathrm{Co}$ ) and the energy gain due to the spin polarization is brought. The origin of the spin polarization can be attributed to the similarity of their environment. This is confirmed by the similarity of their $d$-orbital DOSs. The "band energy" estimated from total DOS shows that the changes of DOS near the Fermi level bring the band-Jahn-Teller-type stabilization of the distorted structure.
\end{abstract}

(Received April 26, 2004; Accepted May 24, 2004)

Keywords: ferromagnetic shape memory alloy, cobalt-nickel-aluminum alloy, equi-atomic composition, linear muffin-tin orbital atomic sphere approximation

\section{Introduction}

The ferromagnetic shape memory alloys (FSMAs) attract a great deal of attention due to the fact that the shape memory effect can be controlled by applying magnetic field in addition to conventional controls by temperature and/or stress.

Recently, Co-Ni-Al alloys have been developed as new FSMAs. ${ }^{1-3)}$ Oikawa et al. $^{1)}$ have investigated Co-Ni-Al alloys and have shown the following facts. (1) A system of ferromagnetic $\beta$ phase $\mathrm{Co}-\mathrm{Ni}-\mathrm{Al}$ alloys with the $B 2$ structure exhibits the shape memory effect. (2) The alloys with the composition range (30-45 at\%)Co-Ni-(27-32 at\%)Al undergo a paramagnetic/ferromagnetic transition as well as a thermoelastic martensitic transformation from the $\beta$ (B2) to the $\beta^{\prime}\left(L 1_{0}\right)$ phase. (3) The Curie and the martensitic start temperatures in the $\beta$ phase can be controlled independently to fall within the range of $120-420 \mathrm{~K}$.

To expand our knowledge of electronic structures and magnetic properties for ferromagnetic shape memory Co-Ni$\mathrm{Al}$ alloys, we have performed first-principles band calculations for the composition of $\mathrm{Co}: \mathrm{Ni}: \mathrm{Al}=1: 1: 1$, i.e., $\left(\mathrm{Co}_{1 / 3^{-}}\right.$ $\left.\mathrm{Ni}_{2 / 3}\right)\left(\mathrm{Co}_{1 / 3} \mathrm{Al}_{2 / 3}\right)$ and $\left(\mathrm{Ni}_{1 / 3} \mathrm{Co}_{2 / 3}\right)\left(\mathrm{Ni}_{1 / 3} \mathrm{Al}_{2 / 3}\right)$. Their electronic structures have been calculated for the supercell structure, including a cubic-tetragonal distortion and a spin polarization.

Band calculations have been performed by the linear muffin-tin orbital atomic sphere approximation (LMTOASA) method ${ }^{4)}$ within a local spin density approximation (LSDA). ${ }^{5,6)}$ The ratio of atomic sphere radii is determined by

\footnotetext{
${ }^{*} 1$ This Paper was Presented at the Spring Meeting of the Japan Institute of Metals, held in Tokyo, on April 1, 2004

${ }^{*}$ Present address: National Center for University Entrance Examinations, Tokyo 153-8501, Japan.
}

trial and error to satisfy the charge-neutrality requirement for each atom. The value is $1.00(\mathrm{Co}): 0.99(\mathrm{Ni}): 1.08(\mathrm{Al})$.

The contents of this paper are as follows. In $\S 2$, we will explain the adopted supercell structure. In $\S 3$, we will show electronic structures and magnetic properties obtained from band calculations and discuss the stabilization of the ferromagnetic state and the distorted structure. This paper comes to a conclusion in $\S 4$.

\section{Model Structure}

We assume the crystal structures of Co-Ni-Al alloys as follows. Figure 1 shows two supercells for the austenite and martensite structure $\left(\mathrm{SC}_{\mathrm{A}}\right.$ and $\left.\mathrm{SC}_{\mathrm{M}}\right)$, both of which have a tetragonal symmetry of the space group $P 4 / \mathrm{mmm}^{7)}$ The austenite supercell $\left(\mathrm{SC}_{\mathrm{A}}\right)$ is composed of three unit cells of the cubic $B 2$ structure, i.e. 6 atoms (4 inequivalent sites) per cell (see Table 2).

In this paper, we will use $a=a_{\mathrm{M}}$ and $c=c_{\mathrm{M}} / 3$, where $a_{\mathrm{M}}$ and $c_{\mathrm{M}}$ denote the lattice constants of the martensite supercell $\left(\mathrm{SC}_{\mathrm{M}}\right)$. If $a_{\mathrm{A}}$ and $c_{\mathrm{A}}$ denote the lattice constants of the $\mathrm{SC}_{\mathrm{A}}$, then $a=\sqrt{2} a_{\mathrm{A}}$ and $c=c_{\mathrm{A}} / 3$. Thus, the $\mathrm{SC}_{\mathrm{M}}$ with $c / a=$ $1 / \sqrt{2}(\approx 0.707)$ is equivalent to the undistorted structure.

Table 1 shows the first and second neighboring sites of each site in the $\mathrm{SC}_{\mathrm{M}}$ with $c / a \neq 1 / \sqrt{2}$. Table 2 shows the occupancies of inequivalent sites in the supercell for $\left(\mathrm{Co}_{1 / 3} \mathrm{Ni}_{2 / 3}\right)\left(\mathrm{Co}_{1 / 3} \mathrm{Al}_{2 / 3}\right)$ and $\left(\mathrm{Ni}_{1 / 3} \mathrm{Co}_{2 / 3}\right)\left(\mathrm{Ni}_{1 / 3} \mathrm{Al}_{2 / 3}\right)$. The models for $\left(\mathrm{Co}_{1 / 3} \mathrm{Ni}_{2 / 3}\right)\left(\mathrm{Co}_{1 / 3} \mathrm{Al}_{2 / 3}\right)$ and $\left(\mathrm{Ni}_{1 / 3} \mathrm{Co}_{2 / 3}\right)\left(\mathrm{Ni}_{1 / 3^{-}}\right.$ $\left.\mathrm{Al}_{2 / 3}\right)$ are named as CNCA and NCNA, respectively.

Before we will show results, we make some comments. Paramagnetic electronic structures of $\mathrm{NiAl}$ and $\mathrm{CoAl}$ have been calculated for the conventional cubic $B 2$ structure and the above-mentioned supercell structures $\left(\mathrm{SC}_{\mathrm{A}}\right.$ and $\left.\mathrm{SC}_{\mathrm{M}}\right)$. Then, we have made sure that there hardly exist differences among those in the points of the total energy per formula unit 


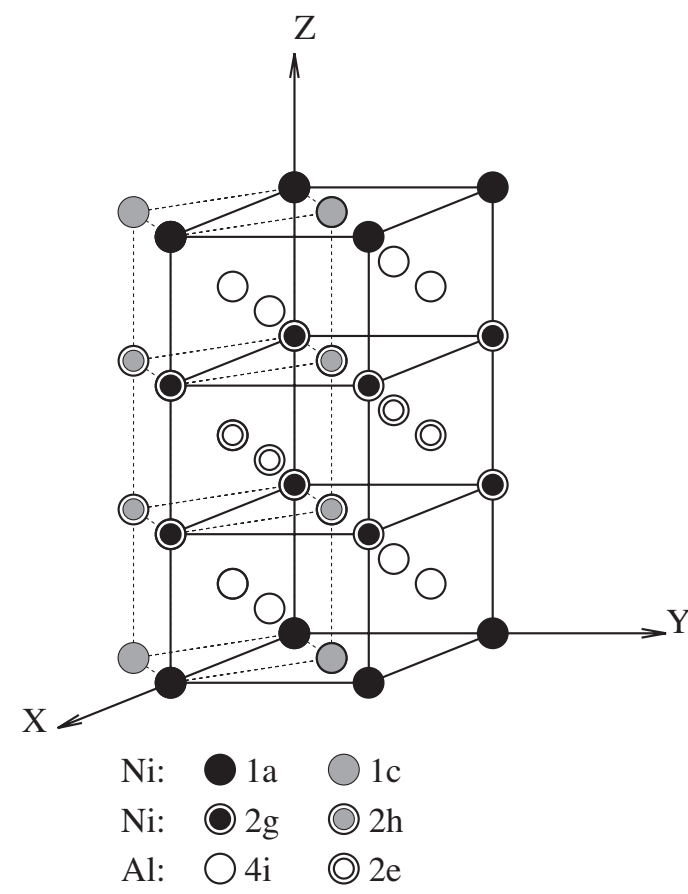

Fig. 1 Supercells corresponding to the martensite phase. The cell showed by the broken line corresponds to the austenite phase.

Table 1 First and second atomic configuration in the $\mathrm{SC}_{\mathrm{M}}$ with $c / a \neq$ $1 / \sqrt{2}$.

\begin{tabular}{ccc}
\hline Site & First neighbor & Second neighbor \\
\hline $1 \mathrm{a}$ & $8(4 \mathrm{i})^{\mathrm{a})}$ & $4(1 \mathrm{c})$ \\
$1 \mathrm{c}$ & $8 \mathrm{i}(4 \mathrm{i})$ & $4(1 \mathrm{a})$ \\
$2 \mathrm{~g}$ & $4(4 \mathrm{i}) 4(2 \mathrm{e})$ & $4(2 \mathrm{~h})$ \\
$2 \mathrm{~h}$ & $4(4 \mathrm{i}) 4(2 \mathrm{e})$ & $4(2 \mathrm{~g})$ \\
$2 \mathrm{e}$ & $4(2 \mathrm{~g}) 4(2 \mathrm{~h})$ & $4(2 \mathrm{e})$ \\
$4 \mathrm{i}$ & $2(1 \mathrm{a}) 2(1 \mathrm{c}) 2(2 \mathrm{~g}) 2(2 \mathrm{~h})$ & $4(4 \mathrm{i})$ \\
\hline
\end{tabular}

a) For example, "8(4i)" means that the atom at the 1a site has 8 atoms at the $4 \mathrm{i}$ site in the first neighbor.

Table 2 Occupancies of inequivalent sites in two supercells, $\mathrm{SC}_{\mathrm{A}}$ and $\mathrm{SC}_{\mathrm{M}}$.

\begin{tabular}{|c|c|c|c|c|c|c|c|}
\hline \multirow{2}{*}{$\begin{array}{l}\text { Name } \\
\mathrm{SC}_{\mathrm{A}}\end{array}$} & \multicolumn{4}{|c|}{ Site and atom } & \multicolumn{3}{|c|}{ Numbers of atom } \\
\hline & $1 \mathrm{a}$ & $2 g$ & $1 d$ & $2 \mathrm{~h}$ & & & \\
\hline $\mathrm{SC}_{\mathrm{M}}$ & $1 \mathrm{a} 1 \mathrm{c}$ & $2 \mathrm{~g} 2 \mathrm{~h}$ & $2 \mathrm{e}$ & $4 \mathrm{i}$ & $\mathrm{Al}$ & $\mathrm{Ni}$ & $\mathrm{Co}$ \\
\hline $\mathrm{CNCA}^{\mathrm{a}}$ & $\mathrm{Co}$ & $\mathrm{Ni}$ & $\mathrm{Co}$ & $\overline{\mathrm{Al}}$ & 4 & 4 & 4 \\
\hline $\mathrm{NCNA}^{\mathrm{b}}$ & $\mathrm{Ni}$ & $\mathrm{Co}$ & $\mathrm{Ni}$ & $\mathrm{Al}$ & 4 & 4 & 4 \\
\hline
\end{tabular}

a) CNCA corresponds to $\left(\mathrm{Co}_{1 / 3} \mathrm{Ni}_{2 / 3}\right)\left(\mathrm{Co}_{1 / 3} \mathrm{Al}_{2 / 3}\right)$.

b) NCNA corresponds to $\left(\mathrm{Ni}_{1 / 3} \mathrm{Co}_{2 / 3}\right)\left(\mathrm{Ni}_{1 / 3} \mathrm{Al}_{2 / 3}\right)$.

(f.u.) and the lattice constant estimated from the minimum of the total energy. This fact may allow us safely to regard the $\mathrm{SC}_{\mathrm{M}}$ with $c / a=1 / \sqrt{2}$ as the austenite structure corresponding to the cubic $B 2$ structure.

\section{Results and Discussion}

\subsection{Total energy, $c / a$ and magnetic moment}

Figure 2 shows the results of total energy $(E)$ vs tetragonality $(c / a)$ for CNCA and NCNA in the paramagnetic (P) and ferromagnetic (F) states, where $E$ is total energy per

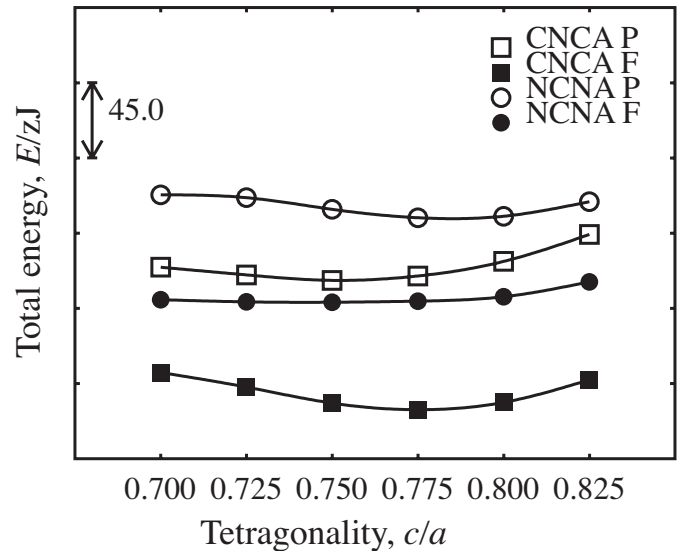

Fig. 2 Total energy per u.c. $(E)$ vs tetragonality $(c / a)$ for CNCA and NCNA in the paramagnetic and ferromagnetic states.

unit cell (u.c.). Here we have done the following procedure. First, for each of the $\mathrm{P}$ and $\mathrm{F}$ sates, we performed total-energy calculations changing the value of $a$ with the fixed value of $c / a=1 / \sqrt{2}$ (corresponding to an undistorted structure) and then determined the lattice constant (or the volume $v_{0}$ ) which minimizes the total energy. Next, we performed total-energy calculations changing the value of $c / a$ with the fixed value of $v_{0}$, and then determined the $c / a$ which minimizes the total energy. The obtained value of $c / a$ are 0.753 and 0.775 (0.784 and 0.743 ) for the $\mathrm{P}$ and $\mathrm{F}$ states of CNCA (NCNA), respectively. The $c / a$ ratio for the $\mathrm{F}$ state of CNCA is about $5 \%$ smaller than the experimental value $(c / a=0.816)$ for 33Co-Ni-28Al. ${ }^{8}$ )

Figure 2 indicates that $\mathrm{CNCA}$ is more appropriate than NCNA because the total energy of CNCA is lower than that of NCNA in both cases of P and F states. Therefore, we pay attention to $\mathrm{CNCA}$ in the following. The results for total energies of CNCA suggest the following transformation in the Co-Ni-Al alloys with the composition of $\mathrm{Co}: \mathrm{Ni}: \mathrm{Al}=$ 1:1:1. A paramagnetic austenite phase transforms into a ferromagnetic martensite phase via a paramagnetic martensite phase on cooling. This type of magnetic and martensitic transformation has been observed in $\mathrm{Co}-\mathrm{Ni}-29 \mathrm{at}_{\%} \mathrm{Al}^{1)}$ and Co-Ni-30 at $\% \mathrm{Al}^{2}{ }^{2)}$ where combinations of magnetic transformation and martensitic transformation are grouped into three types from the results of the composition dependency of the Curie temperature, the martensitic transformation temperature and the austenitic finishing temperature. In order to understand (or discuss) this interesting composition dependency, we must perform band calculations for several compositions. This investigation is in progress. Then, the discussion for the composition dependency will be postponed until the finish of those band calculations.

The obtained magnetic moments on each atomic site are listed in Table 3, where there hardly exist differences in values of the magnetic moments between the 1a and 1c $(2 \mathrm{~g}$ and $2 \mathrm{~h}$ ) sites and therefore one value is listed for the sites.

Table 3 shows that the Co atoms $(\mathrm{Co}[2 \mathrm{e}])$ on the $2 \mathrm{e}$ site, which is occupied by the $\mathrm{Al}$ atoms in the $\mathrm{B2}$-type $\mathrm{NiAl}$ alloy, play an important role in the magnetic transition between the $\mathrm{P}$ and $\mathrm{F}$ states. The $\mathrm{Co}[2 \mathrm{e}]$ atoms carry magnetic moments $1.70 \mu_{\mathrm{B}}$ corresponding to those of fcc Co (hcp Co) and the 
Table 3 Magnetic moments in CNCA.

\begin{tabular}{lcccc}
\hline & \multicolumn{4}{c}{ Moments $\left(\mu_{\mathrm{B}}\right)$} \\
\hline & $1 \mathrm{a} / 1 \mathrm{c}$ & $2 \mathrm{~g} / 2 \mathrm{~h}$ & $2 \mathrm{e}$ & $4 \mathrm{i}$ \\
$c / a=1 / \sqrt{2}$ & -0.071 & 0.177 & 1.645 & -0.009 \\
$c / a=0.775$ & -0.029 & 0.261 & 1.696 & -0.010 \\
\hline
\end{tabular}

energy gain due to the spin polarization is brought. (Our calculated data for fcc Co and hcp Co with $c / a=1.633$ are 1.66 and $1.60 \mu_{\mathrm{B}}$, respectively.) This can be attributed to the similarity of their environment. As shown in Table 1, the $\mathrm{Co}[2 \mathrm{e}]$ atom has $8 \mathrm{Ni}$ and $4 \mathrm{Co}$ atoms in the first and second nearest neighbor, respectively. The distance from $\mathrm{Co}[2 \mathrm{e}]$ to $\mathrm{Co}$ atoms is about $10 \%$ longer than that to the $\mathrm{Ni}$ atom. Thus, the $\mathrm{Co}[2 \mathrm{e}]$ atom has a similar environment to fcc Co or hcp Co. This mainly causes the spin polarization (spin magnetization) of the $\mathrm{Co}[2 \mathrm{e}]$ atom. This fact is also confirmed by the similarity of their $d$-orbital density of states (DOS). (The $d$ orbital DOS of $\mathrm{Co}[2 \mathrm{e}]$ is shown in the next subsection but that of fcc or hep Co is not shown in this paper. The $d$-orbital DOS of fcc Co, for example, can be seen in Ref. 9.).

\subsection{Density of states}

Figure 3 shows total density of states (TDOS) of CNCA in the $\mathrm{P}$ and $\mathrm{F}$ states. In this figure, the TDOSs for undistorted $(c / a=1 / \sqrt{2})$ and distorted $(c / a=0.775)$ cases are shown. Each contribution to the TDOS from the partial DOS (PDOS) of each constituent atom can be understood from Fig. 4, where the symbol Co(d), for example, means the $d$ orbital of the Co atom. In Fig. 4, we do not show the $d$-orbital DOS of the $\mathrm{Co}$ atom on the $1 \mathrm{c}$ site $(\mathrm{Co}[1 \mathrm{c}])$ because its shape is very similar to that of the $\mathrm{Co}$ atom on the 1 a site $(\mathrm{Co}[1 \mathrm{a}])$. The same situation is occurred in case of $d$-orbital DOSs for the $\mathrm{Ni}[2 \mathrm{~g}]$ and $\mathrm{Ni}[2 \mathrm{~h}]$ atoms.

From Fig. 3, we can see the TDOS's change brought from the distortion. Though there are several portions that the change is remarkable, it is particularly important that the change in the $\mathrm{P}$ state between $-0.2 \mathrm{aJ}$ and $E_{\mathrm{F}}$, where $\mathrm{Co}[2 \mathrm{e}]$ and $\mathrm{Ni}[2 \mathrm{~g}]$ atoms have large peaks in their PDOSs as shown

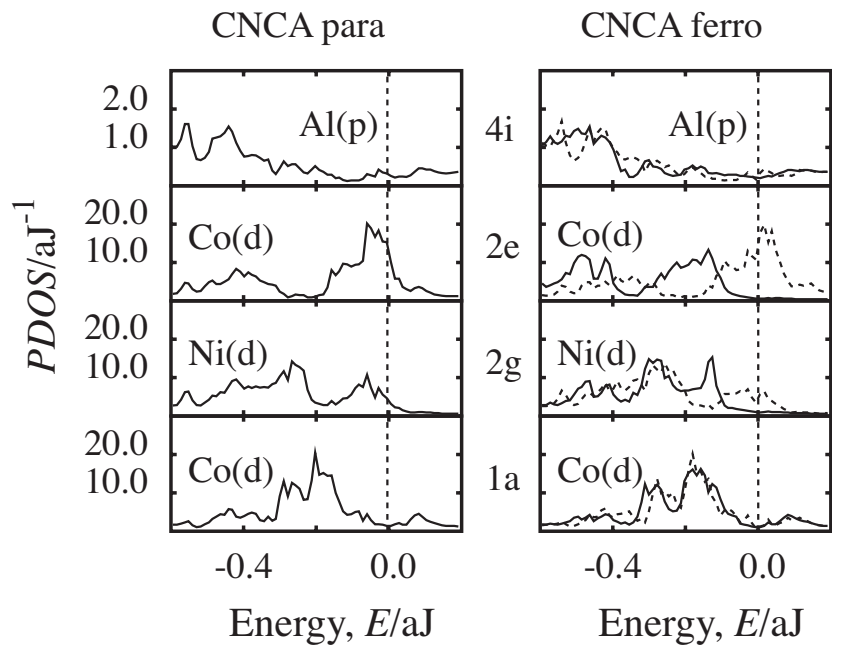

Fig. 4 PDOS (States/(atom spin)) of CNCA in the paramagnetic and ferromagnetic states. PDOSs for majority-spin and minority-spin electrons are shown by the solid and broken lines. The Fermi level is shown by the vertical broken line.

in Fig. 4. (The reason for being important will be explained in the next subsection.)

Figure 4 shows that the $\mathrm{Co}[2 \mathrm{e}]$ atom has a large peak at the Fermi level $\left(E_{\mathrm{F}}\right)$ in the $\mathrm{P}$ state. This fact suggests that the $\mathrm{Co}[2 \mathrm{e}]$ atom carries a magnetic moment due to a spin polarization. This is confirmed by the result shown in Table 3, as already mentioned, and the PDOS of the F state shown in Fig. 4.

\subsection{Band energies}

We have estimated the band energy of each constituent atom from its $P D O S$ in order to quantitatively investigate the PDOS's change brought from the distortion. The band energy $\left(E_{\mathrm{b}}\right)$ is written as $E_{\mathrm{b}}=\int_{-\infty}^{E_{\mathrm{F}}} \epsilon D(\epsilon) d \epsilon$, where $D(\epsilon)$ denotes $P D O S$ at an energy $\epsilon$.

The results for CNCA are shown in Fig. 5, where, "P-P" ("F-F") means the band-energy difference $\left(\Delta E_{\mathrm{b}}\right)$, i.e., $\Delta E_{\mathrm{b}}=E_{\mathrm{b}}($ distorted $)-E_{\mathrm{b}}($ undistorted $)$, for the paramagnetic

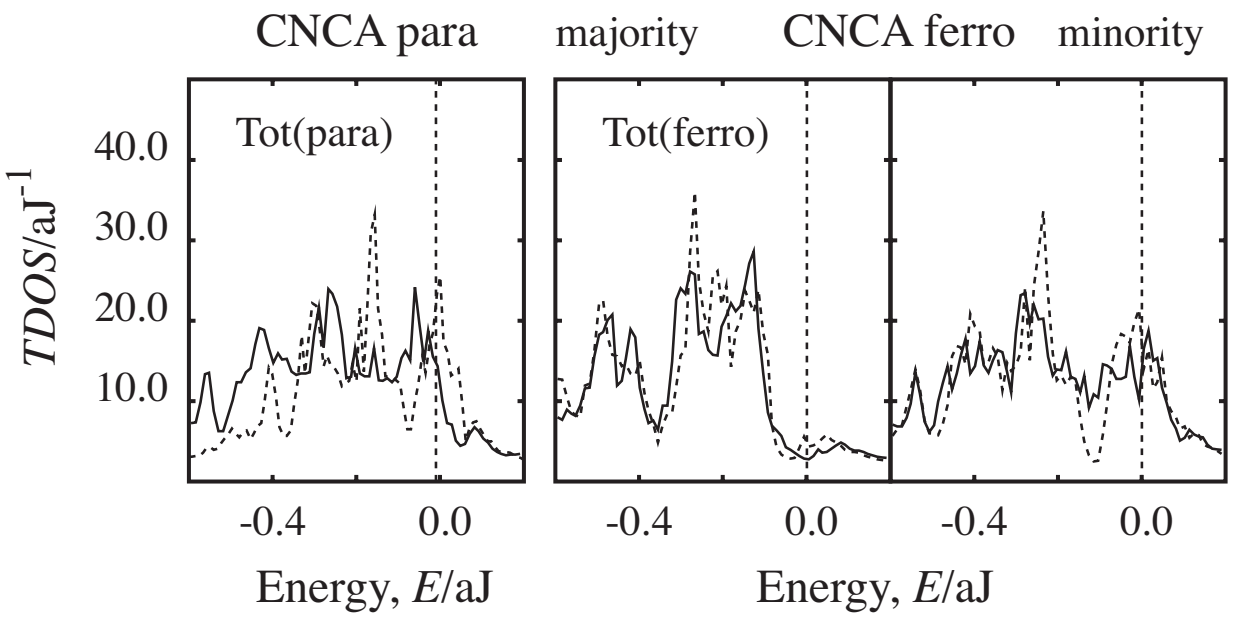

Fig. 3 TDOS (States/(f.u. spin)) of CNCA in the paramagnetic and ferromagnetic states. TDOSs for the undistorted and the distorted structure are shown by the broken and solid lines. The Fermi level for the distorted structure is shown by the vertical broken line. 


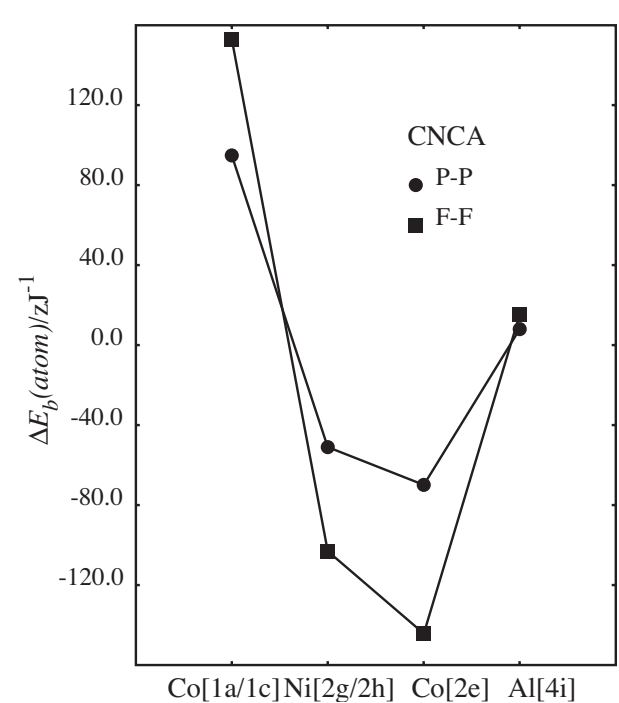

Fig. 5 Band-energy difference per atom $\left(\Delta E_{\mathrm{b}}(\right.$ atom $\left.)\right)$ between the undistorted and the distorted structure for the paramagnetic and the ferromagnetic state.

(ferromagnetic) state. Moreover, the symbol "Co[1a/1c]", for example, means that the value of $\Delta E_{\mathrm{b}}$ is the average of those for the $\mathrm{Co}[1 \mathrm{a}]$ and $\mathrm{Co}[1 \mathrm{c}]$ atoms.

Figure 5 shows that the band energies of $\mathrm{Ni}[2 \mathrm{~g} / 2 \mathrm{~h}]$ and $\mathrm{Co}[2 \mathrm{e}]$ are negative, while that of $\mathrm{Co}[1 \mathrm{a} / 1 \mathrm{c}]$ is positive. The band energy of $\mathrm{Al}[4 \mathrm{i}]$ is positive but negligible. Since the band-energy gain per cell are 0.12 and 0.33 aJ for the $\mathrm{P}$ and $\mathrm{F}$ states, respectively, the band-energy gain of $\mathrm{Ni}[2 \mathrm{~g} / 2 \mathrm{~h}]$ and $\mathrm{Co}[2 \mathrm{e}]$ exceeds the loss of $\mathrm{Co}[1 \mathrm{a} / 1 \mathrm{c}]$ and $\mathrm{Al}[4 \mathrm{i}]$ in both states. The origin of the band-energy gain of the $\mathrm{Ni}[2 \mathrm{~g} / 2 \mathrm{~h}]$ and $\mathrm{Co}[2 \mathrm{e}]$ atoms mainly attributes to the change of their environment, which comes from the transformation from the undistorted to the distorted structure.

The band-energy dependency on energy can be seen in Fig. 6, where enlarged $d$-orbital DOSs near $E_{\mathrm{F}}$ are also shown. It is notable that the band-energy difference per formula unit $(\Delta E(f . u)$.$) becomes zero at an energy \left(E_{0}\right)$ between $-0.2 \mathrm{aJ}$ and $E_{\mathrm{F}}$, and afterward becomes negative in the $\mathrm{P}$ state. This suggests the band-Jahn-Teller effect in the PDOSs of the Co[2e] and $\mathrm{Ni}[2 \mathrm{~g}]$ atoms. That is, the PDOS's peaks near $E_{\mathrm{F}}$ of the $\mathrm{Co}[2 \mathrm{e}]$ and $\mathrm{Ni}[2 \mathrm{~g}]$ atoms are split (or broadened) owing to lowering of the symmetry, when the crystal structure changes from the cubic to the tetragonal structure. This is confirmed by their enlarged $d$-orbital DOSs near $E_{\mathrm{F}}$ shown in Fig. 6. Though the PDOS's peak of the $\mathrm{Co}[1 \mathrm{a}]$ atom is separated from $E_{\mathrm{F}}$, its split or broadened effect also contributes to the above behaviour of $\Delta E(f . u$. $)$ near $E_{\mathrm{F}}$.

\section{Conclusion}

Using a supercell method, we have performed firstprinciples band calculations for the composition of $\mathrm{Co}: \mathrm{Ni}$ : $\mathrm{Al}=1: 1: 1$, i.e., $\left(\mathrm{Co}_{1 / 3} \mathrm{Ni}_{2 / 3}\right)\left(\mathrm{Co}_{1 / 3} \mathrm{Al}_{2 / 3}\right)$ and $\left(\mathrm{Ni}_{1 / 3} \mathrm{Co}_{2 / 3}\right)$ $\left(\mathrm{Ni}_{1 / 3} \mathrm{Al}_{2 / 3}\right)$. The total energy indicates that $\left(\mathrm{Co}_{1 / 3} \mathrm{Ni}_{2 / 3}\right)$ $\left(\mathrm{Co}_{1 / 3} \mathrm{Al}_{2 / 3}\right)$ is more appropriate than $\left(\mathrm{Ni}_{1 / 3} \mathrm{Co}_{2 / 3}\right)\left(\mathrm{Ni}_{1 / 3}-\right.$ $\left.\mathrm{Al}_{2 / 3}\right)$. The result of total energies of the former model

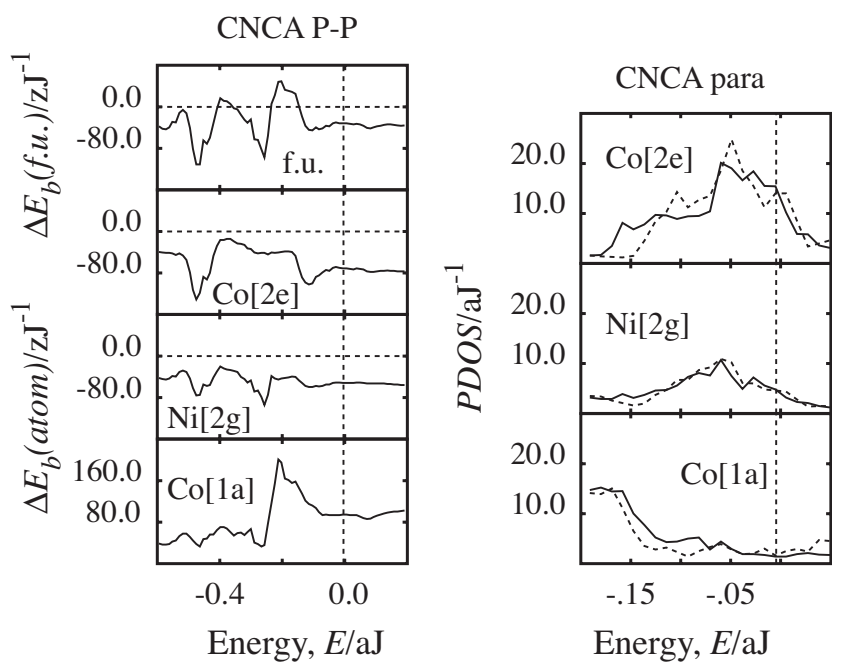

Fig. 6 Band-energy dependency on energy (left) and PDOSs of the undistorted and the distorted structure near the Fermi level (right). In the left, $\Delta E_{\mathrm{b}}($ atom $)$ and $\Delta E_{\mathrm{b}}($ f.u.) show band-energy differences per atom and that per f.u., respectively. In the right, $d$-orbital DOSs for the undistorted and the distorted structure are shown by the broken and solid lines. The Fermi level for the distorted structure is shown by the vertical broken line.

structure (CNCA) suggests the following transformation; A paramagnetic austenite phase transforms into a ferromagnetic martensite phase via a paramagnetic martensite phase on cooling.

The structural transformation in the $\mathrm{P}$ state comes from the band-Jahn-Teller effect. This is reflected in the band energy and the TDOS near $E_{\mathrm{F}}$.

The spin polarization of the $\mathrm{Co}[2 \mathrm{e}]$ atom brings the energy gain and causes the magnetic transition from the paramagnetic to the ferromagnetic state in CNCA.

\section{Acknowledgements}

A part of the present work was supported by the Grant-inaids for Scientific Research from the Ministry of Education, Science, Sports and Culture, Japan.

\section{REFERENCES}

1) K. Oikawa, L. Wulf, T. Iijima, F. Gejima, T. Ohmori, A. Fujita, K. Fukamachi, R. Kainuma and K. Ishida: Appl. Phys. Lett. 79 (2001) 3290-3292.

2) K. Oikawa, T. Ota, F. Gejima, T. Ohmori, R. Kainuma and K. Ishida: Mater. Trans. 42 (2001) 2472-2475.

3) Y. Murakami, D. Shindo, K. Oikawa, R. Kainuma and K. Ishida: Acta Mater. 50 (2002) 2173-2184.

4) O. K. Andersen: Phys. Rev. 12 (1975) 3060.

5) P. Hohenberg and W. Kohn: Phys. Rev. 136 (1964) B864.

6) W. Kohn and L. J. Sham: Phys. Rev. 140 (1965) A1133.

7) T. Hahn (ed.): International Tables for Crystallography, (D. Reidel, Boston, 1983).

8) R. Kainuma, M. Ise, C.-C. Jia, H. Ohtani and K. Ishida: Intermetallics 4 (1996) S151-S158.

9) V. L. Moruzzi, J. F. Janak and A. R. Williams: Calculated Electronic Properties of Metals, (Pergamon Press Inc., 1978). 